\title{
Analysis of flagellar bending in hyperactivated hamster and mouse spermatozoa
}

\author{
F. Aoki ${ }^{1}$, K. Ishida ${ }^{2}$, M. Okuno ${ }^{3}$ and K. Kohmoto ${ }^{1}$ \\ ${ }^{1}$ Department of Animal Breeding, Faculty of Agriculture, University of Tokyo, Bunkyo-ku, Tokyo, Japan; \\ ${ }^{2}$ Department of Urology, Teikyo University School of Medicine, 2-11-1 Koga, Itabashi-ku, Japan; and \\ ${ }^{3}$ Department of Biology, College of Arts and Science, University of Tokyo, Komaba, Meguro-ku, Tokyo, \\ Japan
}

\begin{abstract}
Flagellar bending was analysed using photographs of hyperactivated hamster and mouse spermatozoa. The flagellar waveform consists of several bends; the centre of each bend was therefore located on the flagellum, and the angle of the bend measured. The direction of the bend was determined by using the asymmetry of hook-shaped head to assess the asymmetry of flagellar waveform. The bend that occurred in the same direction as the curve of head was defined as the reverse bend and the bend in the opposite direction as the principal bend. In hamster spermatozoa, flagellar bending was asymmetric to the direction of the reverse bend after incubation for $5 \mathrm{~min}$. After incubation for $4 \mathrm{~h}$ the asymmetry had increased, as the angles of the reverse bends had increased in all regions of the flagellum but the principal bend had not. In mouse spermatozoa incubated for $5 \mathrm{~min}$, flagellar bending was relatively symmetric. In the hyperactivated mouse spermatozoa incubated for $3 \mathrm{~h}$, the angle of the principal bend increased in the distal region and those of the reverse bends increased in almost all regions of the flagellum. Since the increase in the reverse bend was relatively high, flagellar bending became asymmetric to the direction of the reverse bend as in hamster spermatozoa. These increases in asymmetry were also evident in the measurement of the total changes in angular direction between the proximal and distal end of flagella in both species. The increase in asymmetry could provide an explanation for the changes in the motility patterns seen in spermatozoa after the onset of hyperactivation. The mechanism of hyperactivation is discussed in relation to the changes in flagellar bending pattern.
\end{abstract}

\section{Introduction}

Mammalian spermatozoa undergo a change in their pattern of motility during the time of migration in the female genital tract and during incubation in culture in vitro. This change in motility is termed hyperactivation and occurs in association with the process of capacitation (for review, see Yanagimachi, 1981). All hyperactivated spermatozoa swim vigorously but not in a straight direction (Yanagimachi, 1970; Fraser, 1977; Katz et al., 1978; Katz and Yanagimachi, 1980; Suarez et al., 1983; Suarez and Osman, 1987; Morales et al., 1988). However, there are significant differences among species in the patterns of motility of hyperactivated spermatozoa. Hyperactivated mouse spermatozoa swim in an erratic trajectory with frequent rolling (Fraser, 1977; Cooper, 1984; Olds-Clark, 1986); hamster spermatozoa swim in a circular trajectory with rolling at a relatively low frequency (Suarez, 1988); and human spermatozoa swim in a zigzag trajectory with increased lateral motion of the head (Morales et al., 1988).

Revised manuscript received 16 November 1993.
The changes in flagellar bending, responsible for changes in the motility pattern after hyperactivation have not been well examined. The amplitude and asymmetry of flagellar bending increases in hyperactivated spermatozoa (Ishijima and Mohri, 1985; Katz et al., 1986; Suarez, 1988; Suarez et al., 1991), but the relationship between the changes in flagellar bending and changes in motility is not known.

The mechanism that brings about hyperactivation remains unknown. Although there are a number of reports suggesting the involvement of $\mathrm{Ca}^{2+}$ (Yanagimachi and Usui, 1974; Fraser, 1977; Neill and Olds-Clarke, 1987) and cAMP (Morton and Albagli, 1973; Mrsny and Meizel, 1980; Fraser, 1981), there is little information on the mechanism by which these substances change the pattern of flagellar movement. This is because knowledge of flagellar bending in hyperactivated spermatozoa is insufficient to construct a model for the mechanism of hyperactivation.

We therefore investigated the mechanism of hyperactivated motility by analysing the flagellar bending of hyperactivated spermatozoa from mice and hamsters by measuring the bend angles along the whole flagellum. 


\section{Materials and Methods}

\section{Media preparation}

All the chemicals used were purchased from Wako Chemical Inc. (Tokyo), unless otherwise specified, and were of analytical grade.

The medium used for hamster spermatozoa was that developed by Suarez (1988) as this medium maximizes hyperactivation and minimizes the acrosome reaction. It consisted of $110 \mathrm{mmol} \mathrm{NaCl} \mathrm{l}{ }^{-1}, 5.0 \mathrm{mmol} \mathrm{KCl} \mathrm{l^{-1 }}, 2.4 \mathrm{mmol} \mathrm{CaCl} \mathrm{l}^{-1}$, $0.49 \mathrm{mmol} \mathrm{MgCl} 21^{-1}, 0.36 \mathrm{mmol} \mathrm{NaH}{ }_{2} \mathrm{PO}_{4} 1^{-1}, 24.9 \mathrm{mmol}$ $\mathrm{NaHCO}_{3} 1^{-1}, 25.0 \mathrm{mmol}$ Hepes $1^{-1}$ (Nacalai Tesque Inc., Kyoto), $6.25 \mathrm{mmol}$ lactic acid $\mathrm{l}^{-1}, 0.125 \mathrm{mmol}$ sodium pyruvate $1^{-1}, 0.5 \mathrm{mmol}$ hypotaurine $\mathrm{l}^{-1}, 5.0 \mathrm{mmol}$ glucose $\mathrm{I}^{-1}$, $12 \mathrm{mg}$ BSA $\mathrm{ml}^{-1}$ (fatty acid-free Fraction V; Sigma Chemical Inc., St Louis, MO), $100 \mathrm{U}$ penicillin $\mathrm{ml}^{-1}$ and $0.1 \mathrm{mg}$ streptomycin $\mathrm{ml}^{-1}$. The $\mathrm{pH}$ was adjusted to 7.4 with $1 \mathrm{~mol} \mathrm{NaOH} \mathrm{l}{ }^{-1}$.

The medium used for mouse spermatozoa consisted of $136.85 \mathrm{mmol} \mathrm{NaCl} \mathrm{1^{-1 }}, 2.68 \mathrm{mmol} \mathrm{KCl} \mathrm{l^{-1 }}, 1.8 \mathrm{mmol} \mathrm{CaCl}$ $1^{-1}, 0.49 \mathrm{mmol} \mathrm{MgCl}_{2} 1^{-1}, 0.36 \mathrm{mmol} \mathrm{NaH}_{2} \mathrm{PO}_{4} 1^{-1}$, $5.08 \mathrm{mmol} \mathrm{NaHCO} \mathrm{l}^{-1}, 20 \mathrm{mmol}$ Hepes $1^{-1}, 5.55 \mathrm{mmol}$ glucose $\mathrm{I}^{-1}, 0.1 \mathrm{mmol}$ sodium pyruvate $\mathrm{l}^{-1}, 3 \mathrm{mg}$ BSA ml ${ }^{-1}$,

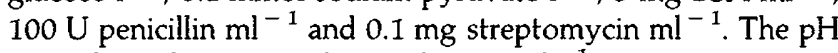

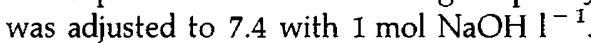

\section{Preparation of spermatozoa}

Male golden hamsters and mice (Jcl:ICR) were killed by cervical dislocation. The cauda epididymides were promptly removed and placed in a plastic Petri dish $(35 \mathrm{~mm} \times 10 \mathrm{~mm})$ containing $5 \mathrm{ml}$ of the respective medium, prewarmed to $37^{\circ} \mathrm{C}$. The distal tubules were either punctured with a 25 -gauge needle in five to ten places (hamsters) or were incised longitudinally by fine scissors (mice), and the fluid and spermatozoa expressed into the medium. The spermatozoa were allowed to disperse into the medium for $5 \mathrm{~min}$, and then passed through a column containing glass beads to prepare samples with high percentages of motile spermatozoa (McGrath et al., 1977). The column consisted of a Pasteur pipette that had had all but $1.0 \mathrm{~cm}$ of the stem removed. One $4.7-5.6 \mathrm{~mm}$ glass bead was placed at the base of the column and $1.5 \mathrm{~g}$ of $0.18-0.25 \mathrm{~mm}$ glass beads (Toshinriko Inc., Tokyo) were packed above the layer bead. After drawing the suspension into the Pasteur pipette several times, $0.5 \mathrm{ml}$ of the spermatozoa suspension was added to the column, followed immediately by $0.8 \mathrm{ml}$ of the medium to elute the motile spermatozoa. The first eluate $(0.3 \mathrm{ml})$ was discarded, the second eluate $(0.5 \mathrm{ml})$ was collected in a $12 \mathrm{~mm} \times 75 \mathrm{~mm}$ glass tube. The resulting suspension was diluted with medium to give a concentration of $2 \times 10^{6}$ spermatozoa $\mathrm{ml}^{-1}$. The procedure for the preparation of the spermatozoa, from release of spermatozoa from the epididymides to the adjustment of sperm concentration, took less than $15 \mathrm{~min}$. The tube was sealed with parafilm and incubated at $37^{\circ} \mathrm{C}$ for $4 \mathrm{~h}$ (hamsters) or $3 \mathrm{~h}$ (mice). These incubation periods are sufficient to induce hyperactivation (Suarez, 1988; Neill and Olds-Clarke, 1987).
Analysis of movement and flagellar bending of spermatozoa

The hamster and mouse spermatozoa were observed under a phase contrast microscope with a heated stage at $37^{\circ} \mathrm{C}$. Samples used for the observation of spermatozoa were prepared as follows. At each time point of the incubation $(5 \mathrm{~min}$ and $4 \mathrm{~h}$ for hamster spermatozoa, and $5 \mathrm{~min}$ and $3 \mathrm{~h}$ for mouse spermatozoa) $12 \mu \mathrm{l}$ aliquots were withdrawn from the sperm suspensions, placed on a glass slide prewarmed to $37^{\circ} \mathrm{C}$, and covered with a $18 \mathrm{~mm} \times 24 \mathrm{~mm}$ cover slip to a depth of $28 \mu \mathrm{m}$. The edges of cover slips were covered with a synthetic mounting agent, Diatex ${ }^{\circledR}$ (polyvinyl acetal: Matsunami Inc., Tokyo) to prevent evaporation. Slides and coverslips had been coated with poly-glutamine-lysine (6:4: Sigma) to minimize sticking of the spermatozoa to the surface of the glass (Stephens et al., 1981). Five successive frames of photographs were then immediately taken, at a rate of 2.5 frames $\mathrm{s}^{-1}$ at an exposure of $1 / 2000 \mathrm{~s}$ (magnification: $\times 25$ for hamster spermatozoa and $\times 37.5$ for mouse spermatozoa). In each experiment, 3-4 fields were randomly selected. The photographic negatives were printed at a magnification of $\times 5$ and the prints were magnified at $\times 1.45$ by copying machine.

The movement characteristics and flagellar bending of spermatozoa were analysed using the successive frames of photographs. Spermatozoa that did not appear on all five frames were excluded from the analysis.

In the analysis of movement characteristics, the swimming trajectories of spermatozoa were determined by tracing the sequential positions of head-midpiece junctions on to a sheet of paper. Two types of swimming speeds were measured. The curvilinear velocity $(V c)$ was determined as the sum of the distance between head-midpiece junctions on successive frames per unit time. The net velocity $(V n)$ was determined from the straight-line distance between the initial and final points of the trajectory. The linear index $(L I)$, a measure of the linearity of the swimming trajectory, was derived by dividing the net velocity by the curvilinear velocity (Suarez, 1988). Some spermatozoa were immotile or swam slowly; spermatozoa with curvilinear velocities of less than $40 \mu \mathrm{m} \mathrm{s}{ }^{-1}$ were therefore omitted from the determination of linearity and from further analysis for flagellar bending.

In the analysis of the bending waves of flagella, the bend angles and the centre of each bend were determined (Fig. I). Since the bending waves consist of circular arcs connected with straight regions (Brokaw and Wright, 1963; Brokaw, 1965), lines were drawn at a tangent to the adjacent arcs to represent the straight regions effectively. The angle between the lines on either side of an arc was measured with a protractor. The direction of each bend was determined according to the direction of the hook-shaped projection of the head as described by Woolley (1977). The bend that occurred in the same direction as the curve of head projection was defined as the reverse bend (R-bend) and the bend in the opposite direction was the principal bend (P-bend). Spermatozoa were excluded from the analysis when the head direction could not be determined. The centre of bend on the flagellum was determined as the point interrupting the bisector of the angle between the lines drawn on either side of the bend. The centre of the bend on the flagellum was located by measuring the distance of the centre of bend $(\mathrm{C})$ from the head-midpiece 


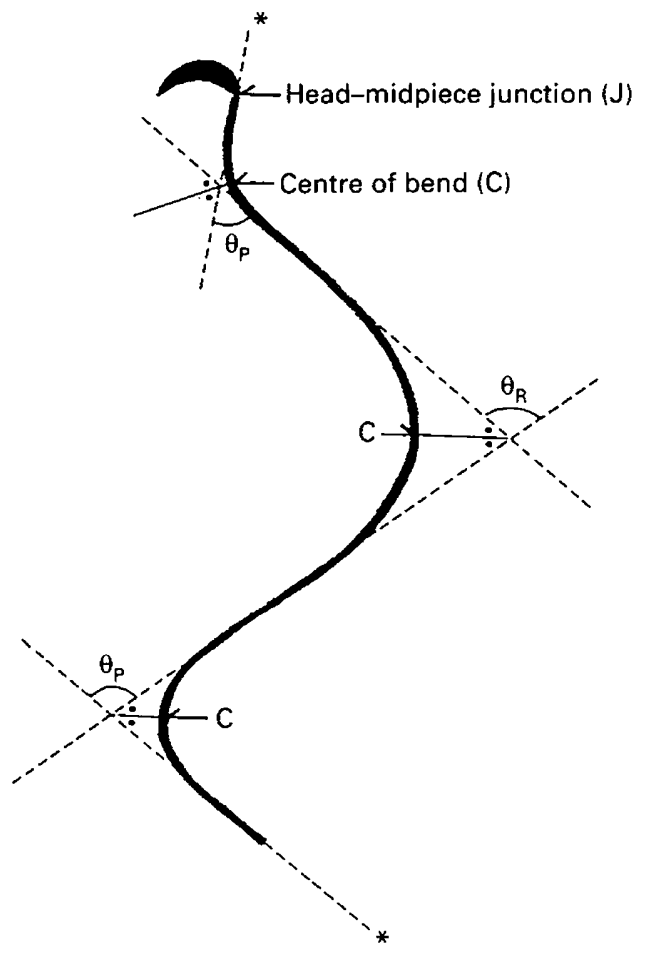

Fig. 1. Analysis of flagellar bending of hamster and mouse spermatozoa. Lines were drawn at a tangent to the bend and the angles $(\theta)$ between the lines measured. Bends in the same direction as the curve of the hook-shaped head were defined as reverse bends (R-bends) and those in the opposite direction as principal bends (P-bends). The centre of each bend on the flagellum was determined as the point interrupting the bisector of the angle between the lines drawn on either side of the bend. The distance of the centre of the bend $(C)$ from the head-midpiece junction (J) was measured and designated as C-J distance. Data on the bend angles were grouped in accordance with the direction (P-bend or R-bend) and the $\mathrm{C}-\mathrm{J}$ distances, and were then averaged to give the values, $\theta_{P}$ and $\theta_{R}$ for $P$-bends and R-bends, respectively. The total change in angular direction between the proximal and distal end of flagellum $\left(\theta_{\text {tip }}\right)$, was also measured. This was determined by measuring the angles between the dashed lines ending in *.

junction (J) by using a curvi-meter (Koizumi Inc., Tokyo) and designated as the C-J distance. C-J distances were grouped in ranges of $22 \mu \mathrm{m}$ for hamster spermatozoa and in ranges of
$15 \mu \mathrm{m}$ for mouse spermatozoa and the average value of each $\mathrm{C}-\mathrm{J}$ distance was taken as the representative value. Data on the bend angles were grouped according to the direction (P-bend or R-bend) and the $\mathrm{C}-\mathrm{J}$ distances, and were then averaged to give the values, $\theta_{\mathrm{P}}$ and $\theta_{\mathrm{R}}$ for the P-bend and R-bend, respectively.

The degree of asymmetry of the flagellum was assessed by measuring the tip bend $\left(\theta_{\text {tip }}\right)$; this is the total change in angular direction between the proximal and distal ends of the flagellum (Gibbons and Gibbons, 1980) (Fig. 1). A bend in the same direction as the head projection was given a positive value and that in the opposite direction a negative value.

\section{Results}

\section{Analysis of motility of hamster spermatozoa}

The motility of hamster spermatozoa was analysed after incubations of $5 \mathrm{~min}$ and $4 \mathrm{~h}$. After incubation for $5 \mathrm{~min}$, the spermatozoa swam in a plane without rolling and in a circular trajectory, in which the hook-shaped projection of the head was always directed into the circle. Similar characteristics of movement were observed after incubation for $4 \mathrm{~h}$, but the circular trajectory became much smaller in most spermatozoa; this characteristic of hyperactivated movement was as described by Suarez (1988). This decrease in diameter of the trajectory after hyperactivation significantly reduced the linear index $(P<0.05)$, as the net velocity was reduced even though the curvilinear velocity remained almost unchanged (Table 1).

\section{Analysis of flagellar bending of hamster spermatozoa}

On the photographs of hamster spermatozoa, a common characteristic was found for both incubation periods (Fig. 2a,b). A small P-bend occurred as the first bend in the proximal region of flagella, and a large $R$-bend as the second bend in the middle region and various sizes of $\mathrm{P}$ - and R-bends in the distal region. The $\mathrm{R}$-bend in the middle region became more acute after incubation for $4 \mathrm{~h}$. This observation was consistent with the results for bend angles. Bend angles, measured separately for P-bends $\left(\theta_{\mathrm{P}}\right)$ and R-bends $\left(\theta_{\mathrm{R}}\right)$, were plotted against $\mathrm{C}-\mathrm{J}$ distance. The values of $\theta_{\mathrm{P}}$ showed no appreciable difference at most of the $\mathrm{C}-\mathrm{J}$ distances for spermatozoa incubated for $5 \mathrm{~min}$

Table 1. Movement characteristics of hyperactivated spermatozoa from mice and hamsters

\begin{tabular}{lccrc}
\hline Animal & Incubation time & $V_{\mathrm{c}}\left(\mu \mathrm{m} \mathrm{s}^{-1}\right)$ & $V_{\mathrm{n}}\left(\mu \mathrm{m} \mathrm{s}^{-1}\right)$ & Linear index \\
\hline Hamster & $5 \mathrm{~min}$ & $123.1 \pm 28.5$ & $101.6 \pm 20.7^{\mathrm{a}}$ & $0.84 \pm 0.03^{\mathrm{b}}$ \\
& $4 \mathrm{~h}$ & $127.2 \pm 30.9$ & $71.1 \pm 5.9^{\mathrm{a}}$ & $0.66 \pm 0.17^{\mathrm{b}}$ \\
Mouse & $5 \mathrm{~min}$ & $112.2 \pm 13.6^{\mathrm{c}}$ & $109.5 \pm 13.8^{\mathrm{d}}$ & $0.98 \pm 0.01^{\mathrm{e}}$ \\
& $3 \mathrm{~h}$ & $83.6 \pm 1.9^{\mathrm{c}}$ & $64.4 \pm 5.7^{\mathrm{d}}$ & $0.76 \pm 0.06^{\mathrm{e}}$
\end{tabular}

Values are means \pm SD of five (hamster) and four (mouse) independent experiments. In each experiment, seven to fifteen spermatozoa were examined. Values with the same superscript are significantly different (Student's test (ab.e: $P<0.05$ and ${ }^{c .0}: P<0.01$ ). $V_{c}$ : curvilinear velocity, $V_{n}$ : net velocity, Linear index: $V_{n}: V_{c}$. 

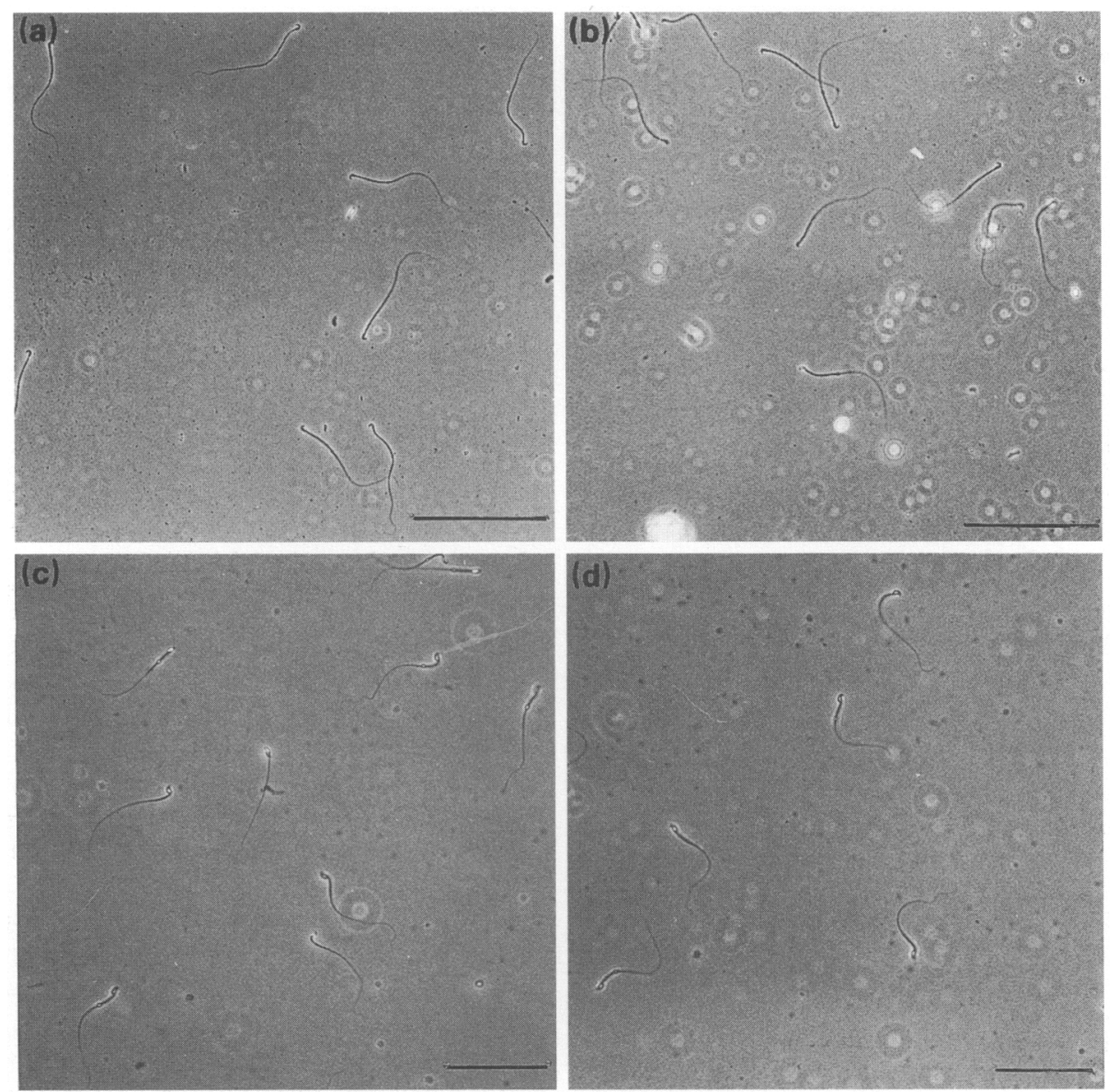

Fig. 2. Phase-contrast micrographs of spermatozoa from hamsters $(a, b)$ and mice (c,d) incubated for $5 \mathrm{~min}(\mathrm{a}, \mathrm{c}), 4 \mathrm{~h}(\mathrm{~b})$ and $3 \mathrm{~h}(\mathrm{~d})$. Scale bars represent $200 \mu \mathrm{m}(\mathrm{a}, \mathrm{b})$ and $100 \mu \mathrm{m}(\mathrm{c}, \mathrm{d})$.

or $4 \mathrm{~h}$, whereas the values of $\theta_{\mathrm{R}}$ were significantly higher $(P<0.01)$ in spermatozoa incubated for $4 \mathrm{~h}$ than for $5 \mathrm{~min}$ at almost all of the $\mathrm{C}-\mathrm{J}$ distances (Fig. 3).

The degree of asymmetry was determined as the value $\theta_{R}-\theta_{P}$ and plotted against C $-\mathrm{J}$ distance (Fig. 4). In spermatozoa incubated for $5 \mathrm{~min}, \theta_{\mathrm{R}}-\theta_{\mathrm{P}}$ was positive in all regions of the flagellum except in the proximal region, indicating that flagellar bending was asymmetric to the direction of the R-bend. After incubation for $4 \mathrm{~h}$, the degree of asymmetry increased because $\theta_{\mathbb{R}}$ increased at almost all the $C-J$ distances.

This increase in the degree of asymmetry was also shown in the measurement of $\theta_{\text {tip }}$. The values of $\theta_{\text {tip }}$ in the same phase of the waves on the flagellum were compared by grouping data on $\theta_{\text {tip }}$ in accordance with the direction and the $\mathrm{C}-\mathrm{J}$ distance of the second bend, and were averaged in each group $\left(\bar{\theta}_{\mathrm{tip}}\right)$. Since the large second bend was observed in almost all spermatozoa, the $\mathrm{C}-\mathrm{J}$ distances of the second bend were used as indices to determine the phase of the wave. Thus, $\bar{\theta}_{\text {tip }}$ in each group was regarded as representative of $\theta_{\text {tip }}$ in each phase of the waves and the maximum and minimum values of $\bar{\theta}_{\text {tip }}$ among each group as those values of $\theta_{\text {tip }}$ in a representative cycle of the wave (Table 2). In spermatozoa incubated for $5 \mathrm{~min}$, even the minimum value of $\vec{\theta}_{\text {tip }}$ was positive, indicating that bending was already asymmetric to the direction of the $\mathrm{R}$-bend. After incubation for $4 \mathrm{~h}$, maximum and minimum values of $\bar{\theta}_{\text {tip }}$ increased, indicating an increase in the degree of asymmetry.

\section{Analysis of motility of mouse spermatozoa}

Although mouse spermatozoa appeared to swim in almost straight paths after incubation for $5 \mathrm{~min}$, more close observation revealed that they turned and switchbacked with regular rotation. After incubation for $3 \mathrm{~h}$, they were hyperactivated and most swam with irregular turning and on an erratic trajectory. The linear indices of these spermatozoa were significantly lower than those of spermatozoa incubated for $5 \min (P<0.05)$ (Table 1$)$.

\section{Analysis of flagellar bending of mouse spermatozoa}

The flagellar bending was analysed after incubation for $5 \mathrm{~min}$ and $3 \mathrm{~h}$ in mouse spermatozoa. As with hamster spermatozoa, a small P-bend, a large R-bend, and P- and R-bends were observed in the proximal, middle, and distal regions of flagellum, respectively, in most spermatozoa (Fig. 2c,d). After 


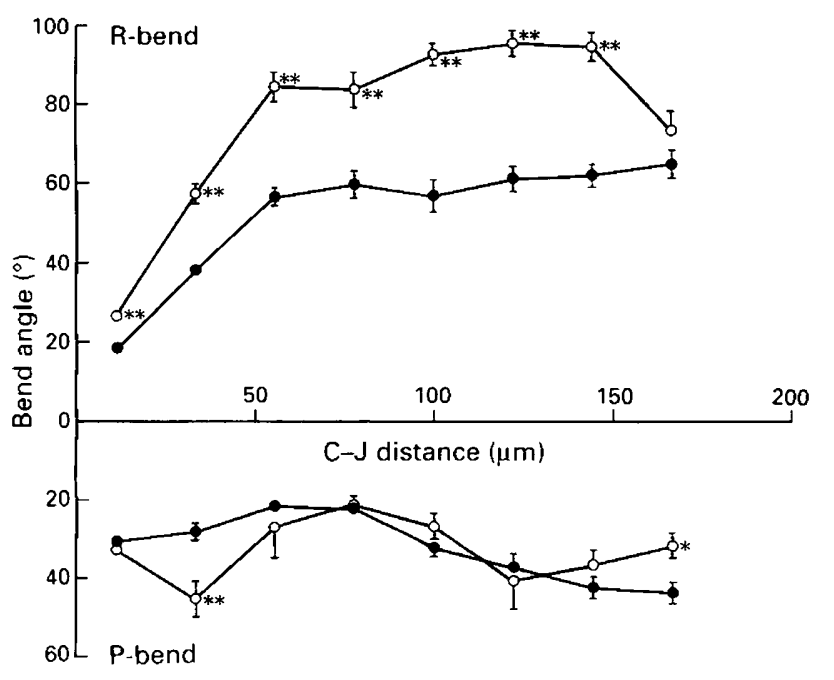

Fig. 3. Changes in bend angles of flagella of hamster spermatozoa after the onset of hyperactivation. The distance of the centre of the bend $(\mathrm{Cc})$ from the head-midpiece junction $(\mathrm{J})(\mathrm{C}-\mathrm{J}$ distance) and angle of each bend on flagella were determined from successive frames of photographs of motile spermatozoa. Data on bend angles were grouped in accordance with the direction of the bends (R-bends: in same direction as the curve of the hook-shaped head and P-bends: in the opposite direction) and with $\mathrm{C}-\mathrm{J}$ distances, and were averaged in each group. The $\mathrm{C}-\mathrm{J}$ distances were grouped in each $22 \mu \mathrm{m}$ range. Spermatozoa were incubated for (•) $5 \mathrm{~min}$ and (o) $4 \mathrm{~h}$. Bars represent SEM. The numbers of data were 44-107 and 34-141 in R- and P-bend in the spermatozoa incubated for $5 \mathrm{~min}$ and $39-89$ and $17-145$ in $\mathrm{R}$ - and P-bend in the spermatozoa incubated for $4 \mathrm{~h}$, respectively. * Significantly different from the corresponding value in spermatozoa incubated for $5 \mathrm{~min}(P<0.05)$; $* * P<0.01$. Results are from five independent experiments.

incubation for $3 \mathrm{~h}$, the P-bend in the proximal region became more obtuse, whereas the large R-bend in the middle region became more acute.

Values of $\theta_{\mathrm{R}}$ increased significantly after incubation for $3 \mathrm{~h}$ at most C-J distances as seen in hamster spermatozoa (Fig. 5). However, the values of $\theta_{\mathrm{P}}$ for mouse spermatozoa increased in the distal region of flagellum. Although the angles of P-bends increased after $3 \mathrm{~h}$ incubation, the asymmetry to the direction of the R-bend increased as in hamster spermatozoa. As the degree of increase in the angles of R-bends was higher than that of $\mathrm{P}$-bends, the values of $\theta_{\mathrm{R}}-\theta_{\mathrm{P}}$ increased at almost all the $\mathrm{C}-\mathrm{J}$ distances after incubation for $3 \mathrm{~h}$ (Fig. 6).

The measurement of $\theta_{\text {tip }}$ also showed that the flagellar bending of mouse spermatozoa became asymmetric to the direction of R-bend after incubation for $3 \mathrm{~h}$ (Table 2). In spermatozoa incubated for $5 \mathrm{~min}$, bending was relatively symmetric, since the maximum and minimum of $\bar{\theta}_{\text {tip }}$ took positive and negative values, respectively, and absolute values were close. After incubation for $3 \mathrm{~h}$, maximum and minimum values increased and the minimum value became close to zero.

\section{Discussion}

We have demonstrated quantitatively in mouse and hamster spermatozoa that after hyperactivation the angle of the R-bend

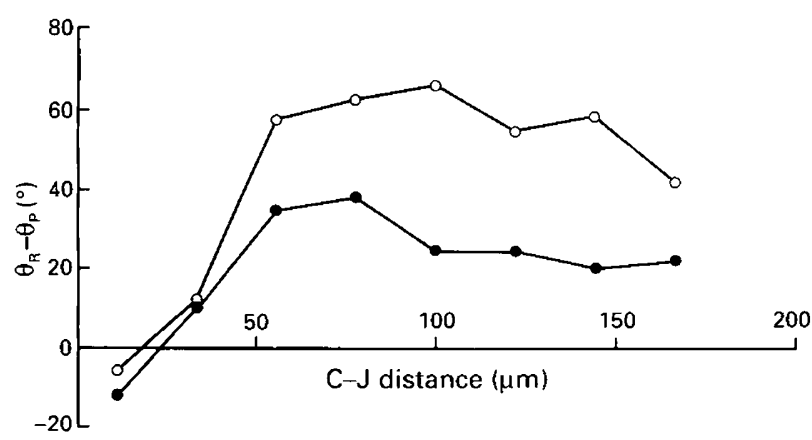

Fig. 4. Changes in the degree of asymmetry of waveform of flagella of hamster spermatozoa after the onset of hyperactivation. The distance of the centre of the bend $(\mathrm{C})$ from the head-midpiece junction (J) (C-J distance) and the angles of the bends on the flagellum were determined. The angles were averaged for each $\mathrm{C}-\mathrm{J}$ distance (grouped in each $22 \mu \mathrm{m}$ range). The value of $\theta_{R}-\theta_{P}$ was determined subtracting the averaged P-bend angle $\left(\theta_{\mathrm{P}}\right)$ from that of R-bend $\left(\theta_{\mathrm{R}}\right)$ where $R$-bends are those in the same direction as the curve of the hook-shaped head and $\mathrm{P}$-bends are those in the opposite direction. Values of $\theta_{R}-\theta_{P}$ from spermatozoa incubated for ( 5 min and (o) $4 \mathrm{~h}$.

increased and that the flagellar bending became asymmetric. An increase in the P-bend was detected in mouse but not in hamster spermatozoa. Nevertheless, the asymmetry to the direction of the R-bend increased in both species, as the changes in the $\theta_{\mathrm{P}}$ were relatively small. The increase in asymmetry was also detected in the measurement of tip-bend angle.

This increase in asymmetry might explain the changes in the motility pattern seen after hyperactivation in mouse and hamster spermatozoa, even though the movement patterns of spermatozoa are quite different in these species. In hamster spermatozoa, flagellar bending was already asymmetric to the direction of R-bend before the onset of hyperactivation. When hamster spermatozoa swim in a single plane without rolling, they would swim in a curve and produce a circular trajectory, with their hook-shaped heads directed into the circles. After the onset of hyperactivation, the asymmetry to R-bend increased, which could lead to spermatozoa swimming in a smaller circle. In mice, the spermatozoa rolled frequently. Before the onset of hyperactivation, the asymmetry of flagellar bending was relatively low and spermatozoa would swim in a slightly curved path. The direction of the curved path seems to coincide with that of the hook-shaped head since the large bend occurring in the middle region of the flagellum is slightly asymmetric to the direction of the R-bend. As the spermatozoa rotate and the direction of the curved path alters, the sequences of such a slightly curved path would produce an almost straight trajectory. In hyperactivated spermatozoa, the increase in asymmetry would make the curved path more acute. As the spermatozoa rotate, the direction of the acute curves is altered, producing an erratic trajectory. Thus, the increase of asymmetry could explain the changes in motility patterns after the onset of hyperactivation in both mouse and hamster spermatozoa. It is possible that the increase in bend angle is involved in the active movement seen in hyperactivated spermatozoa.

In this study, spermatozoa were observed in slide preparations with a depth of $28 \mu \mathrm{m}$ to prevent the flagellum from 
Table 2. Total changes in the angular direction between the proximal and distal end of flagella in hyperactivated spermatozoa from hamsters and mice

\begin{tabular}{|c|c|c|c|c|c|}
\hline \multirow[b]{2}{*}{ Animal } & \multirow[b]{2}{*}{ Incubation time } & \multirow{2}{*}{$\begin{array}{c}\text { Number of } \\
\text { spermatozoa examined }\end{array}$} & \multirow[b]{2}{*}{$\theta_{\text {tip }}{ }^{*}$} & \multicolumn{2}{|c|}{$\bar{\theta}_{\text {tip }}{ }^{\dagger}$} \\
\hline & & & & Maximum & Minimum \\
\hline \multirow[t]{2}{*}{ Hamster } & $5 \min$ & 53 & $49.1 \pm 13.9$ & 90.2 & 13.8 \\
\hline & $4 \mathrm{~h}$ & 57 & $103.1 \pm 17.0$ & 145.7 & 67.7 \\
\hline \multirow[t]{2}{*}{ Mouse } & $5 \mathrm{~min}$ & 35 & $-17.4 \pm 11.1$ & 54.8 & -31.4 \\
\hline & $3 \mathrm{~h}$ & 43 & $35.1 \pm 16.7$ & 87.1 & -0.8 \\
\hline
\end{tabular}

The total changes in the angular direction between the proximal and distal end of flagellum $\left(\theta_{\text {tip }}\right)$ and the distance of the centre of the bend $(\mathrm{C})$ from the head-midpiece junction $(\mathrm{J})(\mathrm{C}-\mathrm{J}$ distance) of the second bend were measured from photographs. Changes that occurred in the same direction as the head projection were given positive values and those in the opposite direction negative values. ${ }^{*} \theta_{\text {tip }}$ is expressed as mean $\pm \mathrm{SD}$. ${ }^{\dagger}$ Data on $\theta_{\text {tip }}$ were grouped in accordance with the direction and C-J distance of the second bend, and were averaged in each group $\left(\bar{\theta}_{\text {tip }}\right)$. Maximum and minimum values of $\bar{\theta}_{\text {tip }}$ among the groups were determined.

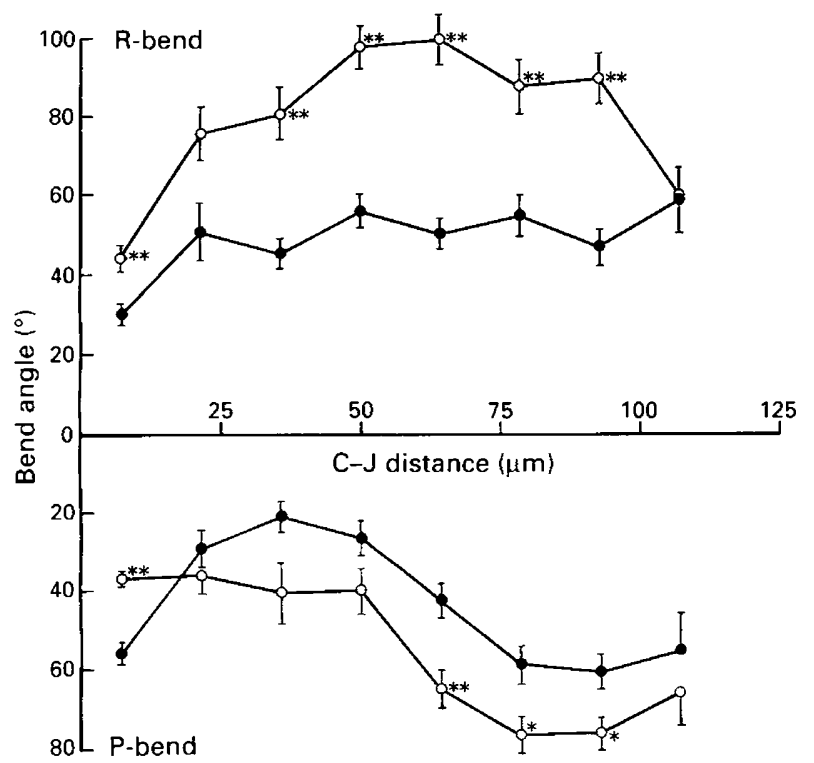

Fig. 5. Changes in bend angles of flagella of mouse spermatozoa after the onset of hyperactivation. The distance of the centre of the bend (C) from the head-midpiece junction (J) (C-J distance) and angle of each bend on flagella was determined from successive frames of photographs of motile spermatozoa. Data on bend angles were grouped in accordance with the direction of bends (R-bends: in same direction as the curve of the hook-shaped head and P-bends: in the opposite direction) and with $\mathrm{C}-\mathrm{J}$ distances, and were averaged in each group. C-J distances were grouped in each $15 \mu \mathrm{m}$ range. Spermatozoa incubated for $(\bullet 5 \mathrm{~min}$ and $(0) 3 \mathrm{~h}$. Bars represent SEM. *Significantly different from the corresponding value in the spermatozoa incubated for $5 \mathrm{~min}(P<0.05) .{ }^{* * P}<0.01$. Results are from four independent experiments.

twisting and to analyse the almost planar flagellar bending correctly. Such a confined space may prevent the threedimensional movement of the flagellum and change the motility pattern. Suarez et al. (1983) reported that the movement parameters of rabbit spermatozoa were affected by the depth of slide preparations; the frequency of rolling differed between spermatozoa in slide preparations with depths of 25 and

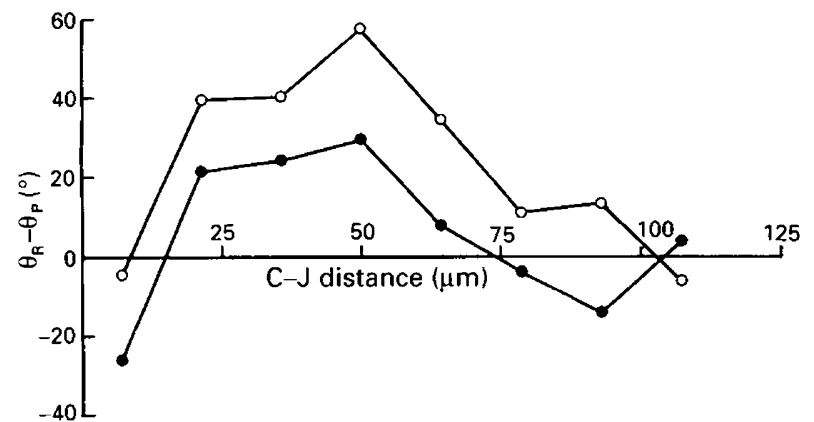

Fig. 6. Changes in the degree of asymmetry of waveform of flagella in mouse spermatozoa after the onset of hyperactivation. The distance of the centre of the bend $(\mathrm{C})$ from the head-midpiece junction $(\mathrm{J})(\mathrm{C}-\mathrm{J}$ distance) and the angles of the bends on the flagellum were determined. The angles were averaged for each $\mathrm{C}-\mathrm{J}$ distance (grouped in each $15 \mu \mathrm{m}$ range). The value of $\theta_{R}-\theta_{P}$ was determined by subtracting the averaged $\mathrm{P}$-bend angle $\left(\theta_{\mathrm{P}}\right)$ from that of $\mathrm{R}$-bend $\left(\theta_{\mathrm{R}}\right)$ where $R$-bends are those in the same direction as the curve of the hook-shaped head and P-bends are those in the opposite direction. Values of $\theta_{R}-\theta_{P}$ from spermatozoa incubated for (•) $5 \mathrm{~min}$ and (o) $3 \mathrm{~h}$.

$100 \mu \mathrm{m}$. However, in their study the velocity and linearity of swimming of spermatozoa did not differ between the two preparations. Indeed, we observed the characteristics of movement similar to those described in previous reports in which a deep chamber was used, before and after the onset of hyperactivation in mouse and hamster spermatozoa.

There are a number of reports suggesting that $\mathrm{Ca}^{2+}$ is involved in hyperactivation: hyperactivation requires $\mathrm{Ca}^{2+}$ in the incubation medium (Yanagimachi and Usui, 1974; Fraser, 1977; Neill and Olds-Clarke, 1987), and hyperactivated motility was induced by the calcium ionophore A23187 in spermatozoa from mice (Fraser, 1982; Cooper, 1984; Suarez et al., 1987), sheep (Shams-Borhan and Harrison, 1981) and guineapigs (Talbot et al., 1976). This hypothesis is supported by our results that hyperactivation increases the asymmetry of sperm motion, since it is well established that $\mathrm{Ca}^{2+}$ affects the asymmetry of flagellar bending. In demembranated spermatozoa from sea urchins and rats, the increase in free $\mathrm{Ca}^{2+}$ concentration induces asymmetry of flagellar bending (Brokaw, 
1979; Gibbons, 1980; Okuno and Brokaw, 1981; Lindemann and Goltz, 1988). This effect of $\mathrm{Ca}^{2+}$ seems to be exerted in all parts of the flagellum. Lindemann and Goltz (1988) reported that all the parts of dissected flagella from rat spermatozoa maintained the shape induced by $\mathrm{Ca}^{2+}$. This finding is consistent with the results reported here that the asymmetry in hyperactivated spermatozoa was observed in almost all the parts of the flagellum.

The changes in flagellar bending observed in hyperactivated spermatozoa do not seem to be induced only by $\mathrm{Ca}^{2+}$. Brokaw (1979) showed that in demembranated sea urchin spermatozoa the increased angle in one direction was compensated for by a decreased angle in the other direction, keeping the mean bend angle constant as the $\mathrm{Ca}^{2+}$ concentration changed. However, such changes did not occur in hyperactivated spermatozoa. Although the angle of the R-bend increased in all regions of the flagellum in hamster and mouse hyperactivated spermatozoa, the decrease in the P-bend occurred only in the distal region in hamster spermatozoa and in the proximal region in mouse spermatozoa. In mouse spermatozoa, increases in the P-bend were in the distal region. It is possible that changes that occur in the mechanisms regulating the amplitude of $\mathrm{P}$ - and $\mathrm{R}$-bends always occur simultaneously and to the same extent. This mechanism might be controlled by a substance, such as ATP or CAMP, which is required for the active sliding of microtubules. This hypothesis also explains the differences observed between the changes in the P-bend between mouse and hamster spermatozoa. In hamster spermatozoa, the mechanism controlling changes in asymmetry might be dominant, whereas in mouse spermatozoa, that controlling amplitude might be.

\section{References}

Brokaw CJ (1965) Non-sinusoidal bending waves of sperm flagella Joumal of Experimental Biology 43 155-169

Brokaw CJ (1979) Calcium-induced asymmetrical beating of tritondemembranated sea urchin sperm flagella Journal of Cell Biology 82 401-411

Brokaw Cl and Wright L (1963) Bending waves of the posterior flagellum of ceratium Science 142 1169-1170

Cooper TG (1984) The onset and maintenance of hyperactivated motility of spermatozoa from the mouse Gamete Research 9 55-74

Fraser LR (1977) Motility patterns in mouse spermatozoa before and after capacitation Journal of Experimental Zoology 202 439-444

Fraser LR (1981) Dibutyryl cyclic AMP decreases capacitation time in vitro in mouse spermatozoa Journal of Reproduction and Fertility 62 63-72

Fraser LR (1982) $\mathrm{Ca}^{2+}$ is required for mouse sperm capacitation and fertilization in vitro Journal of Andrology 3 412-410

Gibbons BH (1980) Intermittent swimming in live sea urchin sperm Journal of Cell Biology 84 1-12

Gibbons BH and Gibbons IR (1980) Calcium-induced quiescence in reactivated sea urchin sperm Journal of Cell Biology 84 13-27

Ishijima S and Mohri H (1985) A quantitative description of flagellar movement in golden hamster spermatozoa Journal of Experimental Biology 114 463-475

Katz DF and Yanagimachi R (1980) Movement characteristics of hamster spermatozoa within the oviduct Biology of Reproduction 22 759-764
Katz DF, Cherr GN and Lambert H (1986) The evolution of hamster sperm motility during capacitation and interaction with the ovum vestments in vitro Gamete Research 14 333-346

Katz DF, Yanagimachi R and Dresdner RD (1978) Movement characteristics and power output of guinea-pig and hamster spermatozoa in relation to activation Journal of Reproduction and Fertility 52 167-172

Lindemann CB and Goltz JS (1988) Calcium regulation of flagellar curvature and swimming pattern in Triton X-100-extracted rat sperm Cell Motility and the Cytoskeleton 10 420-431

McGrath J, Hillman N and Nadijcka M (1977) Separation of dead and live mouse spermatozoa Developmental Biology 61 114-117

Morales P, Overstreet JW and Katz DF (1988) Changes in human sperm motion during capacitation in vitro Journal of Reproduction and Fertility 83 119-128

Morton B and Albagli L (1973) Modification of hamster sperm adenyl cyclase by capacitation in vitro Biochemical and Biophysical Research Communications 50 697-703

Mrsny RL and Meizel S (1980) Evidence suggesting a role for cyclic nucleotides in acrosome reactions of hamster sperm in vitro Joumal of Experimental Zoology 211 153-157

Neill JM and Olds-Clarke P (1987) A computer-assisted assay for mouse sperm hyperactivation demonstrates that bicarbonate but not bovine serum albumin is required Gamete Research 18 121-140

Okuno M and Brokaw CJ (1981) Calcium-induced in form of demembranated sea urchin sperm flagella immobilized by vanadate Cell Motility 1 349-362

Olds-Clarke P (1986) Motility characteristics of sperm from the uterus and oviducts of female mice after mating to congenic males differing in sperm transport and fertility Biology of Reproduction 34 453-467

Shams-Borhan G and Harrison RAP (1981) Production, characterization, and use of ionophore-induced, calcium-dependent acrosome reaction in ram spermatozoa Gamete Research 4 407-432

Stephens DT, Acott TS and Hoskins DD (1981) A cautionary note on the determination of forward motility protein activity with bovine epididymal spermatozoa Biology of Reproduction 25 945-949

Suarez SS (1988) Hamster sperm motility transformation during development of hyperactivation in vitro and epididymal maturation Gamete Research 19 $51-65$

Suarez SS and Osman RA (1987) Initiation of hyperactivated flagellar bending in mouse sperm within the female reproductive tract Biology of Reproduction 36 1191-1198

Suarez SS, Katz DF and Overstreet JW (1983) Movement characteristics and acrosomal status of rabbit spermatozoa recovered at the site and time of fertilization Biology of Reproduction 29 1277-1287

Suarez SS, Vincenti L and Ceglia MW (1987) Hyperactivated motility induced in mouse sperm by calcium ionophore A23187 is reversible Journal of Experimental Zoology 244 331-336

Suarez SS, Katz DF, Owen DH, Andrew JB and Powell RL (1991) Evidence for the function of hyperactivated motility in sperm Biology of Reproduction 44 375-381

Talbot P, Summers RG, Hylander BL, Keough EM and Franklin LE (1976) The role of calcium in the acrosome reaction: an analysis using ionophore A23187 Journal of Experimental Zoology 198 383-392

Woolley DM (1977) Evidence for 'twisted plane' undulations in golden hamster sperm tails Journal of Cell Biology $\mathbf{7 5} 851-865$

Yanagimachi R (1970) The movement of golden hamster spermatozoa before and after capacitation Joumal of Reproduction and Fertility 23 193-196

Yanagimachi R (1981) Mechanisms of fertilization in mammals. In Fertilization and Embryonic Development in vitro, pp 81-182. Eds L Mastroianni and JD Biggers. Plenum Press, New York

Yanagimachi R and Usui N (1974) Calcium dependence of the acrosome reaction and activation of guinea pig spermatozoa Experimental Cell Research 89 161-174 